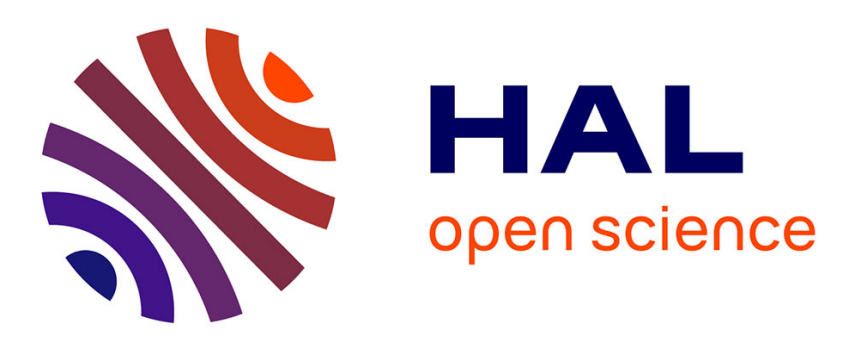

\title{
Handling negative patterns for fast single-pixel lifetime imaging
}

Antonio Lorente Mur, Marien Ochoa, Jérémy E Cohen, Xavier Intes, Nicolas Ducros

\section{- To cite this version:}

Antonio Lorente Mur, Marien Ochoa, Jérémy E Cohen, Xavier Intes, Nicolas Ducros. Handling negative patterns for fast single-pixel lifetime imaging. 2019 - Molecular-Guided Surgery: Molecules, Devices, and Applications V, Feb 2019, San Francisco, United States. pp.1-10, 10.1117/12.2511123 . hal-02017598v2

\section{HAL Id: hal-02017598 \\ https://hal.science/hal-02017598v2}

Submitted on 11 Mar 2019

HAL is a multi-disciplinary open access archive for the deposit and dissemination of scientific research documents, whether they are published or not. The documents may come from teaching and research institutions in France or abroad, or from public or private research centers.
L'archive ouverte pluridisciplinaire HAL, est destinée au dépôt et à la diffusion de documents scientifiques de niveau recherche, publiés ou non, émanant des établissements d'enseignement et de recherche français ou étrangers, des laboratoires publics ou privés. 


\title{
Handling negative patterns for fast single-pixel lifetime imaging
}

\author{
Antonio Lorente Mur ${ }^{\mathrm{a}}$, Marien Ochoa ${ }^{\mathrm{b}}$, Jérémy E Cohen ${ }^{\mathrm{c}}$, Xavier Intes $^{\mathrm{b}}$, and Nicolas Ducros ${ }^{\mathrm{a}}$ \\ ${ }^{a}$ Univ Lyon, INSA-Lyon, UCB Lyon 1,CNRS, Inserm, CREATIS UMR 5220, U1206, Lyon, France \\ ${ }^{\mathrm{b}}$ Department of Biomedical Engineering, Rensselaer Polytechnic Institute, Troy, New York, USA \\ ${ }^{c}$ Univ Rennes, Inria, CNRS, IRISA, Rennes, France
}

\begin{abstract}
Pattern generalization was proposed recently as an avenue to increase the acquisition speed of single-pixel imaging setups. This approach consists of designing some positive patterns that reproduce the target patterns with negative values through linear combinations. This avoids the typical burden of acquiring the positive and negative parts of each of the target patterns, which doubles the acquisition time. In this study, we consider the generalization of the Daubechies wavelet patterns and compare images reconstructed using our approach and using the regular splitting approach. Overall, the reduction in the number of illumination patterns should facilitate the implementation of compressive hyperspectral lifetime imaging for fluorescence-guided surgery.
\end{abstract}

Keywords: Computational imaging, single-pixel camera, semi-nonnegative matrix factorization, pattern generalization

\section{INTRODUCTION}

Most conventional cameras rely on high resolution arrays of detectors. However, building arrays for sophisticated detectors (e.g., hyperspectral, time resolved) is costly, if feasible. In this case, single-pixel imaging might be a good alternative to conventional imaging, as it only requires a single photodetector. ${ }^{1}$ It is particularly relevant for hyperspectral time-resolved imaging, which can retrieve intensity, lifetime, and FRET occurrence maps in vitro. ${ }^{2,3}$

Single-pixel imaging experimentally measures the inner product between the scene and some modulation patterns, and then it reconstructs the image of the scene computationally. ${ }^{4}$ The modulation patterns are usually taken with some transform basis to benefit from fast reconstruction. Common choices include Fourier, ${ }^{5,6}$ wavelet, ${ }^{7-12}$ and Hadamard ${ }^{13,14}$ basis patterns.

While most reconstruction schemes are based on patterns that have both positive and negative values, spatial light modulators can only implement positive patterns. To deal with this issue, it is common practice to split the patterns into two sets of positive patterns, and then to recombine them to measure the target patterns. ${ }^{15}$ However, the splitting requires two measurements to acquire one target pattern. Recently, a pattern generalization method was introduced to circumvent this issue. ${ }^{16}$ The idea is to find some positive patterns that lead to the target pattern through linear combinations. To do so, we solve a modified semi-positive factorization problem by means of a time-consuming optimization algorithm. This has two drawbacks: real-time implementation is not feasible, and the approximation framework induces a remaining factorization error that can translate into reconstruction artifacts.

In this paper, we show a whole family of analytical solutions of the pattern generalization problem. As in Ref. 16, our solution only requires $(I+1)$ measurements to measure $I$ target patterns. However, contrary to Ref. 16, this is an exact factorization scheme and it has fast implementation.

\section{SINGLE-PIXEL THEORY}

\subsection{Acquisition}

Let $\mathbf{f} \in \mathbb{R}^{N \times 1}$ be an image with $N$ pixels. The power emitted by the light source (e.g., laser, ambient light) is denoted by $N_{0} \in \mathbb{R}_{+}$, where the units are photons per second (ph/s). We denote $\alpha \in \mathbb{R}_{+}$(in $\mathrm{ph} / \mathrm{s}$ ) as the dark current; i.e., the signal read by the single detector when $N_{0}=0 \mathrm{ph} / \mathrm{s}$. The signal $m_{k}$ (in ph) measured by the single detector during the integration time $\Delta t \in \mathbb{R}_{+}$(in s) can be modeled as ${ }^{4,17}$

$$
m_{k}=\left(N_{0} \mathbf{p}_{k}^{\top} \mathbf{f}+\alpha\right) \Delta t
$$


where $\mathbf{p}_{k} \in \mathbb{R}_{+}^{N \times 1}$ is a spatial light modulator (SLM) pattern, and $\mathbf{f}$ and $\mathbf{p}$ are normalized; i.e., $\mathbf{f} \in[0,1]^{N \times 1}$ and $\mathbf{p}_{k} \in[0,1]^{N \times 1}$. Let $\mathbf{P}=\left(\mathbf{p}_{1}, \ldots, \mathbf{p}_{K}\right)^{\top} \in \mathbb{R}_{+}^{K \times N}$ be the matrix that contains the sequence of $K$ SLM patterns $\mathbf{p}_{k}$. The measurement vector $\mathbf{m}=\left(m_{1}, \ldots, m_{K}\right)^{\top} \in \mathbb{R}_{+}^{K \times 1}$ that regroups the sequence of measurements $m_{k}$ of Equation (1) is hence given by the matrix equation

$$
\mathbf{m}=\left(N_{0} \mathbf{P f}+\alpha \mathbf{1}_{K}\right) \Delta t
$$

where $\mathbf{1}_{K}=(1, \ldots, 1)^{\top} \in \mathbb{R}^{K \times 1}$.

\subsection{Choice for patterns and reconstruction}

Different approaches have been proposed to design a set of patterns $\mathbf{P}$ and to recover the image $\mathbf{f}$ from the measurements m. Most of these have considered patterns with negative values. Patterns are usually chosen in bases such as Fourier, 5,6 discrete cosine transform, ${ }^{18}$ wavelets, ${ }^{7-12}$ and Hadamard ${ }^{13}$ (see Ref. 19 for a comparison), where the image $\mathbf{f}$ is supposed to be a sparse, so that the number of nonzero measurements $K$ will be much smaller than the dimensions of the image $N$.

In addition, it is common to assume that there is no dark current, so that the image formation model classically considered for image restoration is

$$
m^{\mathrm{tar}}=N_{0} \mathbf{q}^{\top} \mathbf{f} \Delta t
$$

where $\mathbf{q} \in \mathbb{R}^{N \times 1}$ is a SLM pattern with positive and negative values. From now on, we will refer to $\mathbf{q}$ (resp. $m^{\text {tar }}$ ) as the target SLM pattern (resp. measurement). We denote $\mathbf{Q}=\left(\mathbf{q}_{1}, \ldots, \mathbf{q}_{I}\right)^{\top} \in \mathbb{R}^{I \times N}$ as the set of $I$ target patterns and $\mathbf{m}^{\text {tar }}=\left(m_{1}^{\text {tar }}, \ldots, m_{I}^{\text {tar }}\right)^{\top}$ as the vector that contains the corresponding set of target measurements. Applying Equation (3) to the collection of patterns $\mathbf{Q}$ leads to the target measurements

$$
\mathbf{m}^{\operatorname{tar}}=N_{0} \mathbf{Q} \mathbf{f} \Delta t
$$

The image can finally be reconstructed by computing the least-squares solution. Assuming again that the image $\mathbf{f}$ admits a sparse representation $\mathbf{m}^{\text {tar }}$, and therefore belongs to the column space of $\mathbf{Q}$, we estimate $\mathbf{f}$ as

$$
\mathbf{f}^{*}=\mathbf{Q}^{\dagger} \mathbf{m}^{\mathrm{tar}}
$$

where $\mathbf{Q}^{\dagger}=\mathbf{Q}^{\top}\left(\mathbf{Q} \mathbf{Q}^{\top}\right)^{-1}$ is the Moore-Penrose generalized inverse of $\mathbf{Q}$. When $\mathbf{f}$ is sparse in some basis, $\ell_{1}$-penalized algorithms allows perfect reconstruction of $\mathbf{f}$ from $I \ll N$ patterns. $^{4}$

Unfortunately, the absence of dark current in Equation (4) is unrealistic and the patterns $\mathbf{Q}$ with negative values cannot be physically implemented on a SLM.

\section{IMPLEMENTATION OF NEGATIVE PATTERNS}

\subsection{Splitting method}

One of the most practical ways to handle negative patterns is to split them into two sets of positive patterns $\mathbf{Q}^{+} \in \mathbb{R}_{+}^{I \times N}$ and $\mathbf{Q}^{-} \in \mathbb{R}_{+}^{I \times N}$ such that $\mathbf{Q}=\mathbf{Q}^{+}-\mathbf{Q}^{-}$, where $\mathbf{Q}^{+}$and $\mathbf{Q}^{-}$are chosen as the positive and negative parts of $\mathbf{Q}$; i.e.,

$$
\mathbf{Q}_{i, n}^{+}=\max \left(\mathbf{Q}_{i, n}, 0\right) \quad \text { and } \quad \mathbf{Q}_{i, n}^{-}=\max \left(-\mathbf{Q}_{i, n}, 0\right) \quad \forall(i, n) .
$$

Then we acquire the image with both the positive and negative patterns; i.e.,

$$
\mathbf{m}^{+}=\left(N_{0} \mathbf{Q}^{+} \mathbf{f}+\alpha \mathbf{1}_{K}\right) \Delta t \quad \text { and } \quad \mathbf{m}^{-}=\left(N_{0} \mathbf{Q}^{-} \mathbf{f}+\alpha \mathbf{1}_{K}\right) \Delta t .
$$

Finally, the resulting measurements $\mathbf{m}^{+} \in \mathbb{R}^{I}$ and $\mathbf{m}^{-} \in \mathbb{R}^{I}$ are subtracted to get the target measurements $\mathbf{m}^{\text {tar }}$. Mathematically

$$
\mathbf{m}^{\mathrm{tar}}=\mathbf{m}^{+}-\mathbf{m}^{+} .
$$

This method cancels the impact of the dark current $\alpha$ because by taking the difference of both measurements $\mathbf{m}^{+}$and $\mathbf{m}^{-}$ it can be seen that the dark current is dissipated. Unfortunately, this technique requires $2 I$ measurements to get $I$ target measurements. 


\subsection{Shifting method}

Another approach is to shift the patterns toward positive values and measure additional patterns to recover the direct current (DC) component. Let $\mathbf{Q}^{\text {shift }} \in \mathbb{R}_{+}^{I \times N}$ be the shifted patterns and $\mathbf{Q}^{\mathrm{dc}}$ be the DC patterns, such that $\mathbf{Q}=\mathbf{Q}^{\text {shift }}-\mathbf{Q}^{\mathrm{dc}}$, where $\mathbf{Q}^{\text {shift }}$ and $\mathbf{Q}^{\mathrm{dc}}$ are chosen as

$$
\mathbf{Q}_{i, n}^{\text {shift }}=\mathbf{Q}_{i, n}-\min (\mathbf{Q}) \quad \text { and } \quad \mathbf{Q}_{i, n}^{\mathrm{dc}}=-\min (\mathbf{Q}), \quad \forall(i, n) .
$$

Next, we measure the image for the shifted and DC patterns. Note that as all of the patterns in $\mathbf{Q}^{\mathrm{dc}}$ are the same, we only need to acquire them once. Therefore, in practice, we measure

$$
\mathbf{m}^{\text {shift }}=\left(N_{0} \mathbf{Q}^{\text {shift }} \mathbf{f}+\alpha\right) \Delta t \quad \text { and } \quad m^{\text {dc }}=\left(N_{0} \min (\mathbf{Q})(1, \ldots, 1)^{\top} \mathbf{f}+\alpha\right) \Delta t
$$

Finally, the target measurements are computed as

$$
\mathbf{m}_{i, n}^{\mathrm{tar}}=\mathbf{m}_{i, n}^{\mathrm{shift}}-m^{\mathrm{dc}} \quad \forall(i, n) .
$$

Once again, this method also removes the bias introduced by the dark current $\alpha$. The shifting method only requires $I+1$ measurements to get $I$ target measurements. However, it is very sensitive to noise and has limited practical interest, as will be shown in Section 5.

\subsection{Pattern generalization}

Recently, pattern generalization was introduced as a generalization of the splitting and shifting approaches. ${ }^{16}$ As depicted in the pipeline of Fig. 1, pattern generalization starts by seeking a collection of $K$ positive patterns $\mathbf{P} \in \mathbb{R}_{+}^{K \times N}$ such that

$$
\left\{\begin{array}{l}
\mathbf{Q}=\mathbf{T P} \\
\mathbf{P} \geq 0 \\
\mathbf{T} \mathbf{1}_{I}=\mathbf{0}_{I}
\end{array}\right.
$$

where $\mathbf{P} \geq 0$ is shorthand for $\mathbf{P}_{k, n} \geq 0, \forall(k, n), \mathbf{T} \in \mathbb{R}^{I \times K}$ is a transformation matrix, $\mathbf{0}_{I}=(0, \ldots, 0)^{\top} \in \mathbb{R}^{I \times 1}$. As the patterns $\mathbf{P}$ are positive, they can be physically implemented (see Equation (2)).

The patterns defined by $\mathbf{P}$ will henceforth be called generalized patterns. Then, the raw measurements $\mathbf{m}$ are measured by uploading the positive patterns $\mathbf{P}$ into the single-pixel camera. Finally, the target measurements $\mathbf{m}^{\text {tar }}$ of Equation (4) are obtained as

$$
\mathbf{m}^{\mathrm{tar}}=\mathbf{T m}
$$

Note that due to the conditions $\mathbf{T} \mathbf{1}_{I}=\mathbf{0}_{I}$, the target measurements $\mathbf{m}^{\mathrm{tar}}$ are not biased by the dark current $\alpha$ that corrupts the raw measurements $\mathbf{m}$.

As shown in Ref. 16, Equation (12) can be solved numerically for the case $K=I+1$. However, this approach leads to inexact solutions; i.e., $\mathbf{Q} \approx \mathbf{T P}$ and $\mathbf{T} \mathbf{1}_{I} \approx \mathbf{0}_{I}$. Moreover, this optimization problem is computationally intensive and time consuming, making real-time adaptive methods ${ }^{12}$ very difficult to implement.

\section{EXACT PATTERN GENERALIZATION}

In this section, we show a framework for the exact solutions for the pattern generalization problem, which are fast to compute. ${ }^{20}$ Specifically, we present solutions for which only $K=I+1$ positive patterns are required to acquire $I$ nonpositive target patterns. 


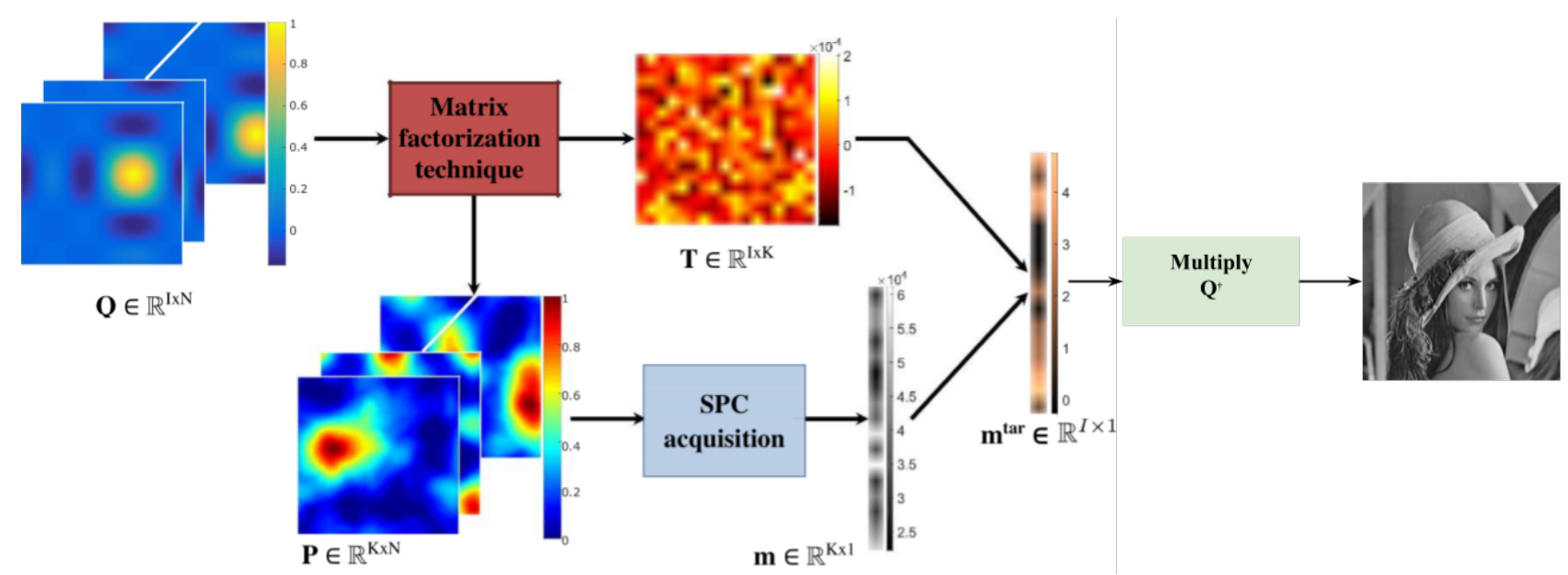

Figure 1: Framework of the pattern generalization method.

\subsection{Shifting variant}

First, let us consider the following matrices:

$$
\mathbf{T}=\left[\begin{array}{ll}
\mathbf{I}_{I} & -\mathbf{1}_{I}
\end{array}\right] \quad \text { and } \quad \mathbf{P}=\left[\begin{array}{c}
\mathbf{Q} \\
\mathbf{0}_{N}^{\top}
\end{array}\right]
$$

where $\mathbf{I}_{I} \in \mathbb{R}^{I \times I}$ is the identity matrix. We can verify that $\mathbf{Q}=\mathbf{T P}$ and $\mathbf{T} \mathbf{1}_{I}=\mathbf{0}_{I}$; i.e., the pair of matrices $\mathbf{T} \in \mathbb{R}^{I \times(I+1)}$ and $\mathbf{P} \in \mathbb{R}^{(I+1) \times N}$ introduced in Equation (14) satisfy the pattern generalization conditions of Equations (12a) and (12c). It is also worth noting that $\mathbf{Q}=\mathbf{T}\left(\mathbf{P}+\left[c_{1} \mathbf{1}_{I} \ldots c_{N} \mathbf{1}_{I}\right]\right)=\mathbf{T P}, \forall\left(c_{1}, \ldots, c_{N}\right)^{\top} \in \mathbb{R}^{N}$, as $\mathbf{T} \mathbf{1}_{I}=\mathbf{0}_{I}$. In other words, given the pair of matrices defined by Equation (14), any matrix obtained by adding a constant $c \in \mathbb{R}$ to any column of $\mathbf{P}$ also satisfies Equation (12a). Choosing $c$ greater than or equal to the lowest value in each column of $\mathbf{Q}$ enables Equation (12b) to be satisfied. In summary, the following pair of matrices exactly solves the generalization problem

$$
\mathbf{T}=\left[\begin{array}{ll}
\mathbf{I}_{I} & -\mathbf{1}_{I}
\end{array}\right] \quad \text { and } \quad \mathbf{P}=\left[\begin{array}{c}
\mathbf{Q} \\
\mathbf{0}_{N}^{\top}
\end{array}\right]-\left[c_{1} \mathbf{1}_{K} \ldots c_{N} \mathbf{1}_{K}\right] .
$$

where $K=I+1$ and $c_{n}=\min _{i}\left(\mathbf{Q}_{i, n}\right)$. This solution is referred to as the shifting variant, as the patterns are shifted so as to make them positive. Contrary to the regular shifting method, each pixel in the patterns is shifted by a different value.

\subsection{Prefactorization method}

In this paragraph, we propose to generalize Equation (15). Assuming that $\mathbf{A} \in \mathbb{R}^{I \times I}$ and $\mathbf{B} \in \mathbb{R}^{I \times N}$ are such that

$$
\mathbf{Q}=\mathbf{A B}
$$

the matrices

$$
\mathbf{T}=\left[\begin{array}{ll}
\mathbf{A} & -\mathbf{A} \mathbf{1}_{I}
\end{array}\right] \quad \text { and } \quad \mathbf{P}=\left[\begin{array}{c}
\mathbf{B} \\
\mathbf{0}_{P}^{\top}
\end{array}\right]
$$

satisfy Equations (12a) and (12c). As for the shifting variant, the positivity conditions of Equation (12b) can be enforced, choosing

$$
\mathbf{T}=\left[\begin{array}{ll}
\mathbf{A} & -\mathbf{A} \mathbf{1}_{I}
\end{array}\right] \quad \text { and } \quad \mathbf{P}=\left[\begin{array}{c}
\mathbf{B} \\
\mathbf{0}_{P}^{\top}
\end{array}\right]-\left[c_{1} \mathbf{1}_{K} \ldots c_{N} \mathbf{1}_{K}\right] .
$$

where $K=I+1$ and $c_{n}=\min _{i}\left(\mathbf{Q}_{i, n}\right)$.

Many different prefactorization variants can be designed depending on the prefactorization that is retained for Equation (16). Natural prefactorization schemes include: 
- Shifting variant. This correspond to the trivial choice

$$
\mathbf{A}=\mathbf{I} \text { and } \mathbf{B}=\mathbf{Q} .
$$

- Singular value decomposition (SVD). Given the SVD $\mathbf{Q}=\mathbf{U} \boldsymbol{\Sigma} \mathbf{V}^{\top}$, we can choose

$$
\mathbf{A}=\mathbf{U} \text { and } \mathbf{B}=\boldsymbol{\Sigma} \mathbf{V}^{\top} .
$$

- Random. Any full rank matrix $\mathbf{C} \in \mathbb{R}^{I \times I}$ can be chosen at random. Therefore, we can choose

$$
\mathbf{A}=\mathbf{C}^{-1} \quad \text { and } \quad \mathbf{B}=\mathbf{C Q} .
$$

\subsection{Batched factorization}

Here, we introduce batched factorization, which relies on splitting the set of patterns $\mathbf{Q}$ into $L$ subsets of patterns; i.e., to consider $\mathbf{Q}^{\top}=\left[\mathbf{Q}_{1}^{\top}, \ldots, \mathbf{Q}_{L}^{\top}\right]$, where $\mathbf{Q}_{\ell} \in \mathbb{R}^{I_{\ell} \times N}$ is the $\ell$-th subset of patterns. This consists of solving the pattern generalization problem for each patterns subset independently

$$
\left\{\begin{array}{l}
\mathbf{Q}_{\ell}=\mathbf{T}_{\ell} \mathbf{P}_{\ell} \\
\mathbf{P}_{\ell} \geq 0 \\
\mathbf{T}_{\ell} \mathbf{1}=\mathbf{0}
\end{array}\right.
$$

where $\mathbf{T}_{\ell} \in \mathbb{R}^{I_{\ell} \times I_{\ell}+1}$ and $\mathbf{P}_{\ell} \in \mathbb{R}^{\left(I_{\ell}+1\right) \times N}$. Therefore, a straightforward solution of the full pattern generalization problem is given by

$$
\mathbf{T}=\operatorname{Diag}\left(\mathbf{T}_{1}, \ldots, \mathbf{T}_{L}\right) \quad \text { and } \quad \mathbf{P}=\operatorname{Diag}\left(\mathbf{P}_{1}, \ldots, \mathbf{P}_{L}\right)
$$

where $\mathbf{T} \in \mathbb{R}^{K \times(K+L)}$ and $K=\sum I_{\ell}$.

\subsection{Numerical simulations}

\section{RESULTS AND DISCUSSION}

The experimental parameters were set to $\alpha=20000$ photons $/ \mathrm{s}, N_{0}=2100$ photons $/ \mathrm{s}$, and $\Delta t=0.1 \mathrm{~s}$. The measurements were corrupted by Poisson noise. The image of the scene is $256 \times 256$, leading to $N=256^{2}$. We considered the well-known Lena image as well as the Jaszczak and bone images provided in the Spirit Toolbox. ${ }^{21}$ All of the simulations were carried out with Daubechies wavelet patterns with four vanishing moments. We performed simulations for varying numbers of target patterns $I$, which led to different compression ratios, as defined in the next paragraph. For each matrix, we will choose $K=I+1$ for the full factorization method. When we use batched factorization, we will choose to have all of the batches of the same size $b_{s}=16\left(\forall i \leq L I_{\ell}=b_{s}\right)$, in which case the total number of required measurements will be $K=I+\left\lceil\frac{I}{b_{s}}\right\rceil$.

We compute matrix $\mathbf{C}$ by uniform sampling over $[0,1]$, and then we orthogonalize this so that we only need to keep one matrix in the random access memory. Pattern generalizations were carried out using all of the methods described in Secs. 3 and 4. Note that the inexact factorization method has always been implemented using batches of size $I_{\ell}=16, \forall \ell$. Indeed, the inexact factorization time without batches takes about $24 \mathrm{~h}$, whereas it only takes a few minutes with batches, on an Intel(R) Core(TM) i5-8400H CPU @ $2.50 \mathrm{GHz}$.

\subsection{Evaluation metrics}

The reconstruction quality will be measured by the peak signal-to-noise ratio (PSNR). The PSNR between the original image $\mathbf{f}$ and the reconstructed image $\mathbf{f}^{*}$ can be defined by

$$
\operatorname{psnr}\left(\mathbf{f}, \mathbf{f}^{*}\right)=10 \log _{10}\left(\frac{d^{2} N}{\left\|\mathbf{f}-\mathbf{f}^{*}\right\|_{2}^{2}}\right)
$$

where $d=256$ is the dynamic range of the image $\mathbf{f}$ and $\|\cdot\|_{2}$ denotes the Euclidean norm.

The acquisition compression ratio is defined by

$$
\mathrm{cr}=100 \times \frac{N-I}{N}
$$

This ranges from $0 \%$ to $100 \%$ (i.e., from no acquisition to no compression). 


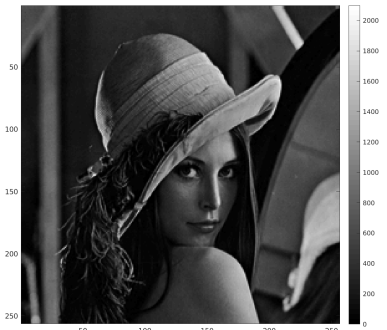

(a)

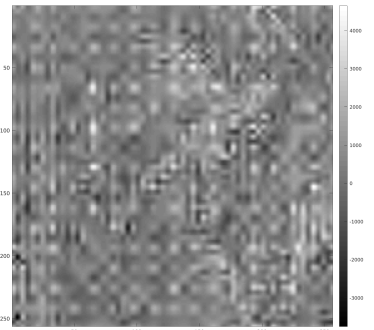

(d)

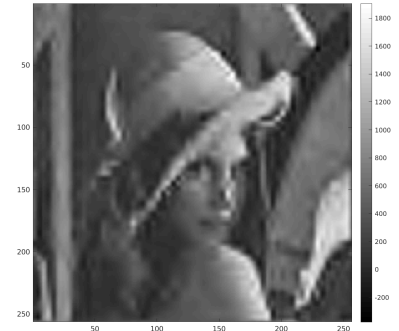

(b)

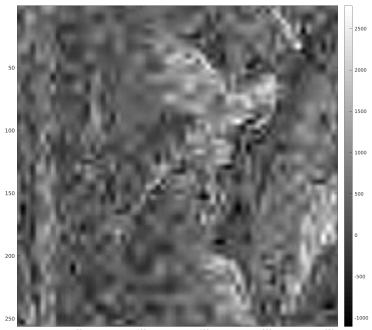

(e)

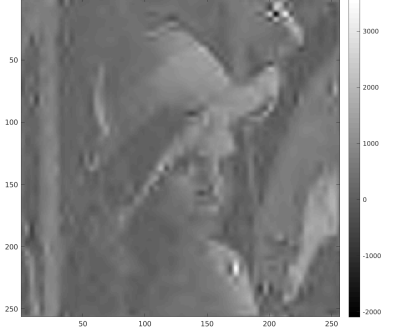

(c)

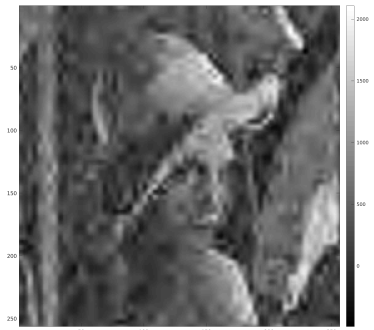

(f)

Figure 2: Reconstructions for the different methods for solving the full pattern generalization problem. The compression rate is set to $97.5 \%$. (a) Ground truth. (b) Split. (c) Inexact. (d) Shift variant. (e) Singular value decomposition. (f) Random. The inexact factorization is computed using batches of size 16, to reduce the computational burden, while no batches are used for the other methods.

Table 1: Peak-to-noise ratios for the reconstructed images for the full pattern generalization problem. Different images and compression ratios are considered across rows, and different methods down columns. SVD, singular value decomposition.

\begin{tabular}{llrrrrrr}
\hline \multirow{2}{*}{ Image } & \multirow{2}{*}{ Compression ratio (\%) } & \multicolumn{3}{c}{ State of the art } & \multicolumn{3}{c}{ Proposed } \\
\cline { 3 - 8 } & & Split & Shift & Inexact & Shift variant & SVD & Random \\
\hline \multirow{3}{*}{ Lena } & 99 & 21.92 & 16.61 & 21.03 & 18.90 & 20.97 & 21.64 \\
& 97.5 & 24.24 & 5.47 & 21.61 & 10.7 & 16.45 & 21.69 \\
& 95 & 24.99 & 2.36 & 21.13 & 7.27 & NaN & 21.91 \\
\hline \multirow{3}{*}{ Jaszczak } & 99 & 19.71 & 17.93 & 19.63 & 18.87 & 19.34 & 19.55 \\
& 97.5 & 24.12 & 11.97 & 22.55 & 16.26 & 20.01 & 22.52 \\
& 95 & 24.51 & 3.24 & 21.67 & 8.19 & 12.25 & 19.25 \\
\hline \multirow{3}{*}{ Bone Image } & 99 & 22.77 & 13.60 & 22.11 & 16.70 & 20.39 & 21.97 \\
& 97.5 & 25.29 & 1.51 & 22.77 & 6.27 & 13.54 & 19.86 \\
& 95 & 25.21 & -1.63 & 19.83 & 3.51 & 9.64 & 20.01 \\
\hline
\end{tabular}

\subsection{Influence of the pattern generalization method}

In Fig. 2, we show visual comparisons between the images that are reconstructed from patterns that originate from different pattern generalization methods. In Table 1, we report the PSNRs of the reconstructions for the different images and compression ratios. Note that the inexact factorization is computed using batches of size 16 , to reduce the computational burden, while no batches are used for the other methods.

It appears that the best reconstruction is obtained by the splitting methods. However, both the inexact factorization and the proposed random exact factorization lead to similar visual quality. The shifting variant and the exact SVD factorization both fail to recover the image.

For decreasing compression ratios, the reconstruction with both the proposed shift variant and the SVD-based factor- 
Table 2: Peak signal-to-noise ratios for the full and batched pattern generalization problems. The batch size was set to $I_{\ell}=16$. Different images and compression ratios are considered across rows, and different methods down columns.

\begin{tabular}{llrrrrrr}
\hline \multirow{2}{*}{ Image } & \multirow{2}{*}{ Compression ratio $(\%)$} & \multicolumn{2}{c}{ Shifting variant } & \multicolumn{2}{c}{ Singular value decomposition } & \multicolumn{2}{c}{ Random } \\
\cline { 3 - 8 } & & Full & Batched & Full & Batched & Full & Batched \\
\hline \multirow{3}{*}{ Lena } & 99 & 18.90 & 21.57 & 20.97 & 21.54 & 21.64 & 21.68 \\
& 97.5 & 10.70 & 22.58 & 16.45 & 22.37 & 21.69 & 23.00 \\
& 95 & 7.27 & 22.32 & NaN & 21.95 & 21.91 & 22.80 \\
\hline \multirow{3}{*}{ Jaszczak } & 99 & 18.87 & 19.47 & 19.34 & 19.50 & 19.55 & 19.56 \\
& 97.5 & 16.26 & 21.82 & 20.01 & 21.82 & 22.52 & 22.52 \\
& 95 & 8.19 & 20.99 & 12.25 & 20.92 & 19.25 & 21.85 \\
\hline \multirow{3}{*}{ Bone image } & 97.5 & 16.7 & 21.80 & 20.39 & 21.73 & 21.97 & 22.09 \\
& 95 & 6.27 & 21.48 & 13.54 & 21.03 & 19.87 & 22.25 \\
& 95 & 3.51 & 20.50 & 9.64 & 20.00 & 20.01 & 21.15 \\
\hline
\end{tabular}

ization dramatically decrease in quality. While the random exact factorization performs better, at low compression ratios it is outperformed by the split method.

\subsection{The interest in batched factorization}

In Fig. 3, we can see that the use of batches greatly improves the accuracy of our reconstruction, particularly when compared to Fig. 2. We can see that the use of batches provides well reconstructed images, even when the shift variant is used.

Table 2 compares the PSNRs computed from the reconstructed images obtained using the different proposed exact factorization patterns with and without the use of batches. In all of our simulated results, batched factorization outperforms full factorization.

Batch factorization increases the number of measurements to be made with respect to full factorization. If $L$ batches are considered, then batch factorization requires $I+L$ measurements, while full factorization only requires $I$ measurements. However, this increase is negligible, as $L \ll I$.

Comparing the results given in Table 2 with those of Table 1, we can see that the proposed random factorization with batches is the best alternative to splitting methods, which provide the best performance, but require doubled the acquisition time ( $I+L$ vs. $2 I$ measurements).

\subsection{Real-time adaptive imaging}

Table 3 compares the different processing times for the different methods. We divided the computation into off-flight computation, which does not require knowledge of the patterns $\mathbf{Q}$, and on-flight computation, which does. This is relevant for adaptive acquisition strategies where the patterns to be measured are determined progressively during the acquisition. ${ }^{12,22}$ Nonadaptive strategies that always measure the same set of patterns can be fully precomputed offline, whatever the retained method for pattern generalization.

It appears that both the proposed shifting variant and the proposed random factorization have relatively low on-flight computation times, which might enable real-time adaptive imaging.

The shift method takes longer with batches because our code for batches is generic and it was not optimized for special cases such as shifting, where the computation could be made easier, so the pre-computation time of the shift method with batches could potentially be improved.

The factorization time is several times faster as the blocks are reduced in size. Also, for highly noisy set-ups, the combination of several very noisy measurements results in a reconstruction where the noise is highly amplified. Hence, reducing the size of the batches might ensure that we are combining less highly noisy measurements. 
Table 3: Computation times for different methods and compression ratios. The measurement time is $K \Delta t$. The pattern generalization precomputation time is denoted by $\Delta t_{\text {off }}$ and the pattern generalization on-flight computation time by $\Delta t_{\mathrm{on}}$. The total acquisition time of an image through an adaptive strategy is $K \Delta t+\Delta t_{\mathrm{on}}$.

\begin{tabular}{|c|c|c|c|c|c|c|c|}
\hline Method & $\begin{array}{c}\text { Offline } \\
\text { computation }\end{array}$ & Batches & Compression ratio (\%) & $I$ & $K$ & $\Delta t_{\text {off }}(\mathrm{s})$ & $\Delta t_{\text {on }}(\mathrm{s})$ \\
\hline \multirow{3}{*}{ Split } & \multirow{3}{*}{ Yes } & \multirow{3}{*}{-} & 99 & 640 & 1280 & 0.7 & - \\
\hline & & & 97.5 & 1638 & 3276 & 1.6 & - \\
\hline & & & 95 & 2867 & 5734 & 2.3 & - \\
\hline \multirow{6}{*}{ Shift } & \multirow{6}{*}{ Yes } & \multirow{4}{*}{ No } & 99 & 640 & 641 & 0.17 & - \\
\hline & & & 97.5 & 1638 & 1639 & 0.4 & - \\
\hline & & & 95 & 2867 & 2868 & 0.8 & - \\
\hline & & & 99 & 640 & 680 & 0.3 & - \\
\hline & & \multirow[t]{2}{*}{ Yes } & 97.5 & 1638 & 1740 & 1 & - \\
\hline & & & 95 & 2867 & 3046 & 2.2 & - \\
\hline \multirow{3}{*}{ Inexact } & \multirow{3}{*}{ No } & \multirow{3}{*}{ Yes } & 99 & 640 & 680 & - & 600 \\
\hline & & & 97.5 & 1638 & 1740 & - & 584 \\
\hline & & & 95 & 2867 & 3046 & - & 925 \\
\hline \multirow{6}{*}{ Shift variant } & \multirow{6}{*}{ Yes } & \multirow{4}{*}{ No } & 99 & 640 & 641 & 0.001 & 0.2 \\
\hline & & & 97.5 & 1638 & 1639 & 0.01 & 0.7 \\
\hline & & & 95 & 2867 & 2868 & 0.06 & 1.2 \\
\hline & & & 99 & 640 & 680 & 0.3 & 0.6 \\
\hline & & \multirow[t]{2}{*}{ Yes } & 97.5 & 1638 & 1740 & 0.7 & 1.5 \\
\hline & & & 95 & 2867 & 3046 & 1.2 & 2.3 \\
\hline \multirow{6}{*}{ Singular value decomposition } & \multirow{6}{*}{ No } & \multirow{4}{*}{ No } & 99 & 640 & 641 & - & 4.5 \\
\hline & & & 97.5 & 1638 & 1639 & - & 22 \\
\hline & & & 95 & 2867 & 2868 & - & 69 \\
\hline & & & 99 & 640 & 680 & - & 2.5 \\
\hline & & \multirow[t]{2}{*}{ Yes } & 97.5 & 1638 & 1740 & - & 6.7 \\
\hline & & & 95 & 2867 & 3046 & - & 11 \\
\hline \multirow{6}{*}{ Random } & \multirow{6}{*}{ Yes } & \multirow{4}{*}{ No } & 99 & 640 & 641 & 0.07 & 0.6 \\
\hline & & & 97.5 & 1638 & 1639 & 1.5 & 2.5 \\
\hline & & & 95 & 2867 & 2868 & 10 & 6.4 \\
\hline & & & 99 & 640 & 680 & 0.3 & 0.6 \\
\hline & & \multirow[t]{2}{*}{ Yes } & 97.5 & 1638 & 1740 & 0.9 & 1.7 \\
\hline & & & 95 & 2867 & 3046 & 1.4 & 2.8 \\
\hline
\end{tabular}




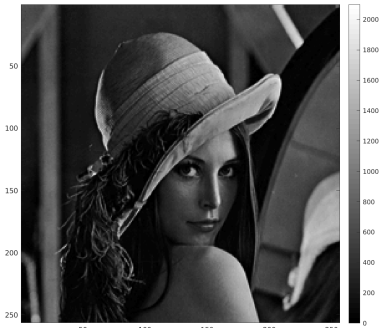

(a)

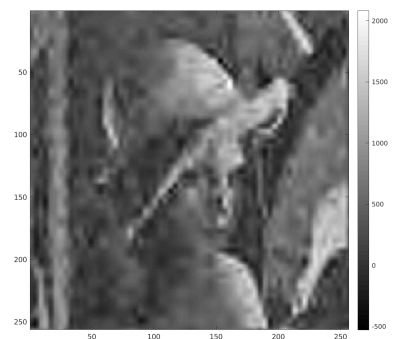

(d)

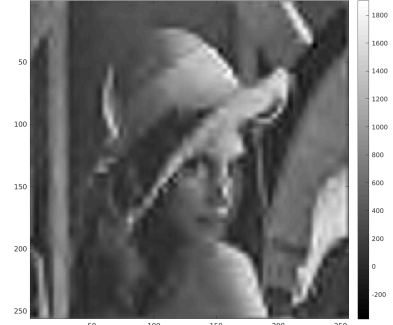

(b)

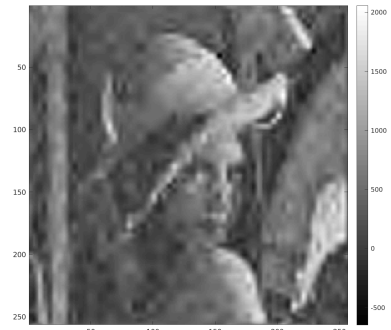

(e)

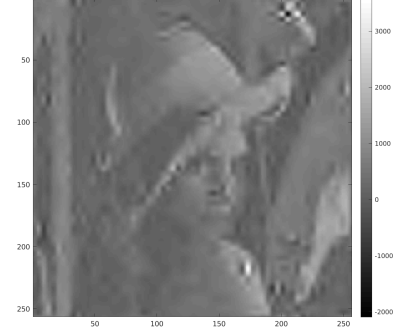

(c)

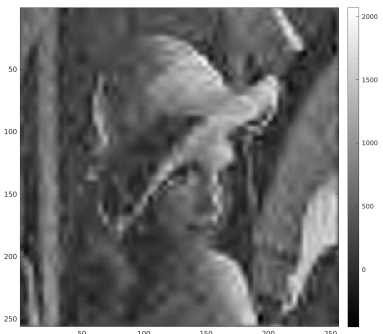

(f)

Figure 3: Comparison of the different pattern generalization methods for a compression rate $\mathrm{cr}=97.5 \%$ and a batch size $I_{\ell}=16$. (a) Ground truth. (b) Split. (c) Inexact. (d) Shift variant. (e) Singular value decomposition. (f) Random.

\section{CONCLUSION AND PERSPECTIVES}

We have defined here a factorization framework that enables reduced acquisition times of single-pixel camera applications, by a factor of two. The use of batches further improves the quality of the acquisition, albeit decreasing the speed-up provided by the pattern generalization method.

The batch method allows us to choose the best compromise between measurement time and measurement quality. The choice of the size of the batch can be from $I$ - the total number of target patterns - to 2 (in which case we get the splitting method exactly).

A certain number of questions still remain. What initialization for our method yields the best results, and why? Empirically the random orthogonal initialization appears to yield the best results, but we have yet to define theoretical conclusive results. Another question would be how to choose the size of the batches that best reflects the compromise sought between speed and accuracy. Furthermore, we are working on the experimental implementation of these methods.

\section{ACKNOWLEDGEMENTS}

This work was supported by the French National Research Agency (ANR), under Grant ANR-17-CE19-0003 (ARMONI Project). It was performed within the framework of the LABEX PRIMES (ANR-11-LABX-0063) of Université de Lyon, within the programme "Investissements d'Avenir" (ANR-11-IDEX-0007) operated by the ANR.

\section{REFERENCES}

[1] Edgar, M. P., Gibson, G. M., and Padgett, M. J., "Principles and prospects for single-pixel imaging," Nature Photonics 13, 13-20 (Jan. 2019).

[2] Pian, Q., Yao, R., Sinsuebphon, N., and Intes, X., "Compressive hyperspectral time-resolved wide-field fluorescence lifetime imaging," Nature Photonics 11, 411 (June 2017).

[3] Rousset, F., Ducros, N., Peyrin, F., Valentini, G., D'Andrea, C., and Farina, A., "Time-resolved multispectral imaging based on an adaptive single-pixel camera," Opt. Express 26, 10550-10558 (Apr 2018). 
[4] Duarte, M., Davenport, M., Takhar, D., Laska, J., Sun, T., Kelly, K., and Baraniuk, R., "Single-pixel imaging via compressive sampling," Signal Processing Magazine, IEEE 25, 83-91 (March 2008).

[5] Zhang, Z., Ma, X., and Zhong, J., "Single-pixel imaging by means of fourier spectrum acquisition," Nature communications 6 (2015).

[6] Zhang, Z. and Zhong, J., "Three-dimensional single-pixel imaging with far fewer measurements than effective image pixels," Opt. Lett. 41, 2497-2500 (Jun 2016).

[7] Deutsch, S., Averbush, A., and Dekel, S., "Adaptive compressed image sensing based on wavelet modeling and direct sampling," in [SAMPTA'09], Laurent Fesquet and Bruno Torrésani, ed., General session (May 2009).

[8] Averbuch, A., Dekel, S., and Deutsch, S., "Adaptive compressed image sensing using dictionaries," SIAM Journal on Imaging Sciences 5(1), 57-89 (2012).

[9] Dai, H., Gu, G., He, W., Liao, F., Zhuang, J., Liu, X., and Chen, Q., "Adaptive compressed sampling based on extended wavelet trees," Appl. Opt. 53, 6619-6628 (Oct 2014).

[10] Hahn, J., Debes, C., Leigsnering, M., and Zoubir, A. M., "Compressive sensing and adaptive direct sampling in hyperspectral imaging," Digital Signal Processing 26, 113 - 126 (2014).

[11] Huo, Y.-R., He, H.-J., Chen, F., and Tai, H.-M., "Adaptive single-pixel imaging based on guided coefficients," J. Opt. Soc. Am. A 34, 39-51 (Jan 2017).

[12] Rousset, F., Ducros, N., Farina, A., Valentini, G., D’Andrea, C., and Peyrin, F., "Adaptive basis scan by wavelet prediction for single-pixel imaging," IEEE Transactions on Computational Imaging 3, 36-46 (March 2017).

[13] Radwell, N., Mitchell, K. J., Gibson, G. M., Edgar, M. P., Bowman, R., and Padgett, M. J., "Single-pixel infrared and visible microscope," Optica 1, 285-289 (Nov 2014).

[14] Gibson, G. M., Sun, B., Edgar, M. P., Phillips, D. B., Hempler, N., Maker, G. T., Malcolm, G. P. A., and Padgett, M. J., "Real-time imaging of methane gas leaks using a single-pixel camera," Opt. Express 25, 2998-3005 (Feb 2017).

[15] Zhang, Z., Wang, X., Zheng, G., and Zhong, J., "Hadamard single-pixel imaging versus fourier single-pixel imaging," Opt. Express 25, 19619-19639 (Aug 2017).

[16] Rousset, F., Peyrin, F., and Ducros, N., "A semi nonnegative matrix factorization technique for pattern generalization in single-pixel imaging," IEEE Transactions on Computational Imaging 4, 284-294 (June 2018).

[17] Takhar, D., Laska, J. N., Wakin, M. B., Duarte, M. F., Baron, D., Sarvotham, S., Kelly, K. F., and Baraniuk, R. G., "A new compressive imaging camera architecture using optical-domain compression," in [in Proc. of Computational Imaging IV at SPIE Electronic Imaging ], 43-52 (2006).

[18] Liu, B.-L., Yang, Z.-H., Liu, X., and Wu, L.-A., "Coloured computational imaging with single-pixel detectors based on a 2d discrete cosine transform," Journal of Modern Optics 64(3), 259-264 (2017).

[19] Ochoa, M., Pian, Q., Yao, R., Ducros, N., and Intes, X., "Assessing patterns for compressive fluorescence lifetime imaging," Opt. Lett. 43, 4370-4373 (Sep 2018).

[20] Gillis, N. and Kumar, A., "Exact and heuristic algorithms for semi-nonnegative matrix factorization," SIAM Journal on Matrix Analysis and Applications 36(4), 1404-1424 (2015).

[21] "Single-pixel image reconstruction toolbox (spirit)." https://www.creatis.insa-lyon.fr/ ducros/ WebPage/spirit.html. Version 2.0, 29th April 2018.

[22] Soldevila, F., Salvador-Balaguer, E., Clemente, P., Tajahuerce, E., and Lancis, J., "High-resolution adaptive imaging with a single photodiode," Scientific Reports (09 2015). 